\title{
Spectrum sharing in cognitive radio networks
}

\author{
Julián Martínez, Cesar Hernandez, Luis Pedraza \\ Technological Faculty, Universidad Distrital Francisco Jose de Caldas, Colombia
}

\begin{tabular}{l} 
Article Info \\
\hline Article history: \\
Received Jan 24, 2020 \\
Revised Jun 6, 2020 \\
Accepted Jun 16, 2020 \\
\hline Keywords: \\
Cognitive radio \\
Cooperative \\
DSA \\
Spectrum sharing \\
TVWS
\end{tabular}

\begin{abstract}
Cognitive radio networks are the next step to tackle scarcity in wireless networks given the increasing demand of radioelectric spectrum where the proposed solution is to share said resource to improve this situation. In the present article, a review of the current state of spectrum sharing in cognitive radio networks. To achieve this purpose, the articles published over the last 4 years on the matter were reviewed including topics such as mobile networks and TV. Some studies and simulations proposed to share the spectrum is shown. The current state of the studies reveals that there has been significant progress in this research area yet it is necessary to continue similar studies and set in motion different schemes.
\end{abstract}

Copyright $(0) 2020$ Institute of Advanced Engineering and Science. All rights reserved.

\section{Corresponding Author:}

Cesar Hernandez,

Technological Faculty,

Universidad Distrital Francisco Jose de Caldas,

Calle 68D Bis A Sur \# 49F - 70, 111941, Bogotá D.C., Colombia.

Email: cahernandezs@udistrital.edu.co

\section{INTRODUCTION}

Currently, wireless communications are part of everyday work activities and the number of devices that connect through these types of networks keeps increasing. This scenario in addition to the large number of mobile devices available lacking wired connections (and even require wireless charging mechanisms) have created the need to improve how these devices connect since networks can become saturated due to the number of connected devices $[1,2]$. Wi-Fi networks are a clear example of this situation which, in spite of having various frequency channels, can suffer from interference in highly-populated areas to the point of generating intermittencies, high latencies and even disable network connection. This is only one of many cases while other issues take place in mobile communications given that licensed frequency bands (used by mobile operators) are not used to their full capacity and leads to white spaces in the acquired channels [2-5].

Therefore, the term of cognitive radio is born [6], defined as a smart radio system that can optimize the use of the radio electric spectrum for licensed and unlicensed users (primary or secondary users). A licensed user refers to any person that pays for a spectrum frequency channel. Usually, the management of this spectrum is taken over by governments and the person paying is the only one that can use it over a certain period of time. Hence, the unlicensed user is a person that does not pay for his use of the spectrum, but could use it in some way while not interfering with the communication of a licensed user $[7,8]$.

These terms lead to dwell on how to share the spectrum and are the basis of the article. The general idea is to assess the progress strived in telecommunications, how simulations and implementations have been carried out and the future work ahead. Hence, different documents have been gathered in which spectrum sharing is discussed over the past 4 years. The present article is distributed as follows. The first section establishes an introduction to different schemes currently in place for spectrum sharing, then some paradigms and dynamic access to the spectrum are discussed, followed by an overview of cooperative spectrum sharing and spectrum sharing in mobile and 5G networks. Finally, before concluding, spectrum sharing in TV bands and business concepts are described that can be useful. 


\section{SPECTRUM SHARING}

Although spectrum sharing may sound simple, it includes various strategies [9] and methods in which it can operate. Hence, before moving on with the work of other authors, it is necessary to learn about the schemes proposed until now to share the spectrum among licensed and unlicensed users.

\subsection{Managed spectrum sharing scheme}

Managed spectrum sharing is one of the studied strategies and consists on a controller in charge of establishing rules regarding the time and place for the spectrum to be shared. Furthermore, it contains all the specifications of the network equipment and technical standards among other data required for connection. The controller uses this information to assign spectrum to users that require part of the spectrum available at the moment [9].

\subsection{Technical schemes of spectrum sharing}

Moving on to spectrum sharing technical schemes, these are divided into mobility, retransmission, routing and recollection of the spectrum. In spectrum mobility [3], the frequency channels of user transmission are switched, in order to guarantee the network performance. This technical scheme establishes that the transmission of the unlicensed user can be suspended at any time to allow the transmission of the licensed user. The latter can free the channel to allow the use of a unlicensed user. This scheme can be divided into two terms, the first one is the spectrum handover $[4,5]$, which defines that if the primary user needs to use the channel used by the secondary user then the latter can move on to another available channel. However, if there are no channels available, the secondary user cannot continue with his transmission. The second term is spectrum management mechanism [5] which is used in ad-hoc cognitive radio networks where there is no central controller in place to carry out the spectrum transfer.

The following technical scheme is the spectrum retransmission [6] which basically helps to increase coverage of secondary users through paths connected with the base station, choosing the one with the lowest response times. The use of this technique increases the performance of the networks and cuts transmission times. For spectrum routing [3], this scheme helps to decide how and when switching between channels must be performed depending on the rival policies and the information regarding channel availability. This switch between user channels is analog to the routing problem in wireless systems. There are several routing protocols that turn the communication of licensed users into a fault-tolerant activity and helps the information recollection nodes to efficiently manage the spectrum.

The final technical scheme is the spectrum recollection [7] which consists on recollecting the energy from cognitive radio devices which are able to turn renewable energies into electricity. The spectrum and energy resources are recollected in an effective manner and managed to save energy. These additional resources are also harnessed through different techniques to save energy. The scheme also increases spectral efficiency in wireless communications In [8], a study was carried out for the joint design of the spectrum detection and power control cooperative time for energy efficiency in the secondary system based on the exchange of hybrid spectrum considering the transmission power, interference limitations, minimum transmission speeds and imperfect spectrum detection. In this case, the simulation results showed the effectiveness of the proposed algorithms.

\subsection{Spectrum commercialization scheme}

The final scheme is based on the commercialization of the spectrum [2] which in turn is divided into commerce as shown in [9] and rent with the example shown in [10]. In the spectrum commerce, the secondary users acquire the rights of the primary user to improve the overall use of the resource. One of the advantages of this commercial scheme is that the secondary user can gain access to the spectrum at a low price. However, this also depends on the primary user since it would act as a monopolizing agent of the allocated spectrum and can offer any price to the secondary user. In terms of spectrum leasing, the licensed user can choose to release some parts of his frequency band in exchange for money or any technical assistance from the secondary user.

\subsection{Dynamic spectrum access (DSA)}

Another important term is dynamic spectrum access (DSA) [11, 12] which a cognitive radio application. This Dynamic Access can be classified into the following strategies: Dynamic Exclusive Use Model, Open Sharing Model and Hierarchical Access Model. The first one is based on regulation policies of the spectrum thus guaranteeing the exclusive use from licensed users and increasing the flexibility and spectral efficiency in the network. This strategy includes the Property Rights of the Spectrum and Dynamic Spectrum Allocation. While the first one consists on leasing the spectrum to licensed users, the second one assigns the spectrum dynamically to secondary users depending on their location, traffic load, among other 
parameters. The open sharing model implies an open sharing policy among users in a spectral region. In [13], it is explained that this open sharing model is not outside of the spectrum regulation yet it does not need to be controlled. Furthermore, the minimum characteristics of the protocol for proper adequation are explained.

Finally, the hierarchical access model also involves two approaches: spectrum overlapping [14] and subjacent spectrum [15]. The first one is focused in the transmission power of the secondary user to cause less interference to licensed users. In the subjacent spectrum, the restrictions take place in the time-space transmission of the secondary users. This indicates where and when the unlicensed user can carry out the transmission. The detection and use of white spaces in the radioelectric spectrum is also an important aspect of the DSA method as seen in studies $[16,17]$. These spaces are defined as the slots in the frequency spectrum that no user is occupying and can be used through DSA techniques. Based on current trends, white spaces can be classified into three paradigms: the subjacent (or underlayer), the overlapped (or overlayer) [18] and woven [17].

In the subjacent paradigm, secondary users transmit in the licensed band while maintaining low power levels to avoid causing interference to the primary users [19-21]. This can be carried out by limiting the power levels of unlicensed users and, as long as the interference from them remains within an acceptable margin, they can transmit simultaneously with the primary user [22-24]. This paradigm also has two divisions which are range/power and hybrid subjacent relay. In the first one, secondary users transmit in the licensed band while their power remains within a specific range to reduce the noise levels in the primary user transmisión [25-27]. In the hybrid subjacent relay, the previous rule is still in place yet the unlicensed user can increase his power levels as long as he supports the communications of the primary user by retransmitting the information for example [9].

In the overlayer paradigm, unlicensed users detect the use of the licensed user spectrum and immediately change their transmission parameters to avoid causing interference. In this case, the transmission of users is simultaneous and can be classified into cooperative and non-cooperative. In cooperative mode, secondary users accept the terms imposed by primary users for spectrum use once they have been admitted. To avoid interference in cooperative use, communication devices with techniques such as FHSS, DSSS and CDMA are available and, while the secondary user is transmitting, he cannot use the primary user code. Therefore, orthogonal codes are used. Furthermore, secondary users can also change the polarization of the radiofrequency antenna to avoid interference by transmitting in the opposite direction to the primary user. The transmission angle can also be switched so that the beam has a specific direction and is not omnidirectional. For non-cooperative overlapping, secondary users transmit independently from white space detection so that there are minimum to no interferences to primary users.

A combination exists between the subjacent and overlapped paradigms known as hybrid access of the spectrum which helps increase the efficiency in the spectrum use through the reduction of handover frequencies. The hybrid scheme has the advantage of switching the spectrum sharing mode between static and dynamic types depending on which offers greater advantages for users that are not using the spectrum [28]. The last woven paradigm in cognitive radio uses the unused spectrum in time, frequency and space/geography when there are no transmissions issued by primary users. In the case that the licensed user begins to transmit again, secondary users must switch to another frequency band available or end their transmission if there are no bands available.

In [27], a comparison was carried out between the subjacent and the woven paradigm in terms of transmission time delays and the data arrival rate. Hence, theoretical and simulation-based calculations were carried out revealing that in the woven paradigm, the delays are lower that in the subjacent paradigm. Furthermore, the same process was also performed for the calculation of the effective transfer rate (throughput) of both schemes. In this case, it was determined that, as the primary user becomes more active, then the transfer rates will become lower for both schemes.

\section{RESULTS AND DISCUSSION}

\subsection{Cooperative spectrum sharing}

As shown in similar schemes, it is necessary that cognitive radio shares the spectrum of the primary user towards secondary users. However, this can also cause interferences in the communications of the licensed user. It is noteworthy to recall that one of the conditions of cognitive radio to share the spectrum is that no interference should occur when the primary user requires a channel for communication. Hence, in [29], a cooperative form of spectrum sharing is proposed in which there is no interference between users and it is based in spatial modulation [30] which uses multiple antennae for communication both for the primary transmitter (PT) and the secondary transmitter (ST). In this case, multiple antennae include a half-duplex configuration to contribute with decoding and forwarding the data of the primary user. 
This spectrum sharing is carried out in two phases. In the first phase, space modulation (SM) is requested by the PT only if the ST is not generating traffic in the channel. In the case of being fulfilled, the bit flow information of the PT is mapped into two sets: the M-ABA modulation based on phase shift keying (M-PSK) over the M-ABA modulation based on quadrature amplitude modulation (M-QAM) which is given in bits and the index or number of antenna. Afterwards, the ST uses the iterative-maximal-ratiocombining (i-MRC) [31] to remove the bitmap of the information blocks transmitted by the PT. In the second phase, the ST sends the primary data through the activation of a single antenna available based on their secondary data which make use of the spatial modulation. The PT data reach the primary receiver (PR) and the secondary receiver (SR). The latter recovers the data by detecting only the transmission indexes of the ST antenna using the i-MRC. With this technique, the interference between primary and secondary systems is null and, hence, it is unnecessary to implement any technique for the cancellation of interferences. This type of spectrum sharing is a convenient option since the primary user does not have to lease his spectrum to gain collaboration from the secondary user and only the i-MRC must be applied to the transmissions. Therefore, the SR can only recover the indexes of the ST antenna from the expected signal.

In the previous study, the sharing environment consists in a primary system (PS) that contains PT and PR and a secondary system (SS) with ST and SR. The proposed system must have a weak direct link between PT and PR, whether it is related to signal fading or deficient condition of the channel. All the communications between PT is carried out by the ST, assuring that the information reaches the PR. For the simulation of this method, parameters such as error probability both in the bit rate and the symbol rate since the transmission between ST and PR must have an accurate estimation of information to avoid errors. Furthermore, the antenna index must also be considered given that it also affects communication. This sharing method included an assessment of the transmission capacity and the probability of cutting the transmission of the PS and SS.

In addition to the interferences that may occur in these systems and spectrum sharing schemes, other aspects such as the efficiency in spectrum use must be factored in both in terms of sharing methods and energy levels. The latter is necessary in cognitive radio systems given the frequent changes that take place in the antenna power, the change of frequency channel, among others. This increases the energy use, thus increasing costs in the support of these systems.

Hence, in addition of the aforementioned schemes explained, the opportunistic spectrum sharing (OSS) [32] and the cooperative spectrum sharing (CSS) [33], which have shown to be capable of offering spectral and energy-related efficiency for transmissions. The OSS allows the secondary user to transmit as long as the primary user does not occupy the spectrum channel. Hence, this scheme has a proper efficiency in the use of the spectrum, but carries no benefit for the primary user. On another note, the CSS includes three phases. In the first one, the primary transmitter sends the signal to the primary receiver and the secondary user. In the second phase, the secondary transmitter sends the signal to the primary receiver. In the third phase, the secondary user uses the spectrum channel to establish communications. Therefore, this communication strategy is beneficial for both primary and secondary users since they can use the channel and cooperate in signal transmission. However, during implementation, there will be more than one secondary user expecting that the spectrum can be used. Furthermore, the CSS model only considers the operation of a single antenna and CSS and MIMO-CSS techniques are restrained in practical applications although MIMO antennae can be implemented with CSS to increase spectrum efficiency. This is evidenced in the studies on game theory and the negotiation of spectrum demand between primary and secondary users.

To compensate the limitations in the OSS scheme, the authors in [34] propose the use of a full-duplex transceiver with the capacity of transmitting and receiving signals in the same frequency which can improve energy and spectral efficiency. This new scheme is called the OSS retransmitter (or ROSS) which works in parallel for the transmission of the primary and secondary user. Therefore, the newly proposed scheme does not require negotiation between users making the ROSS scheme more convenient in practice than the CSS model.

The proposed scenario for cooperative sharing considers classic cognitive radio network with a couple of primary users and a couple of secondary users, who share the same spectrum for transmission. In this case, secondary users have multiple transmission and reception antennae where the first ones cannot surpass the second ones in number. In the ROSS model, when the secondary user wishes to gain access to the spectrum when the communication of the primary user is taking place, he must support the communication of the primary users.

In the spectrum sharing simulations, there is an improvement in the spectral efficiency system ROSS (SE-ROSS) compared to the CSS and MIMO-CSS methods. The SNR for the primary user is equal to $4 \mathrm{~dB}$ and it is expected that the transmission speed of the primary user in both cases increases by a range of $0.2 \mathrm{bit} / \mathrm{s} / \mathrm{Hz}$. The results of this simulation are shown in Figure 1. 

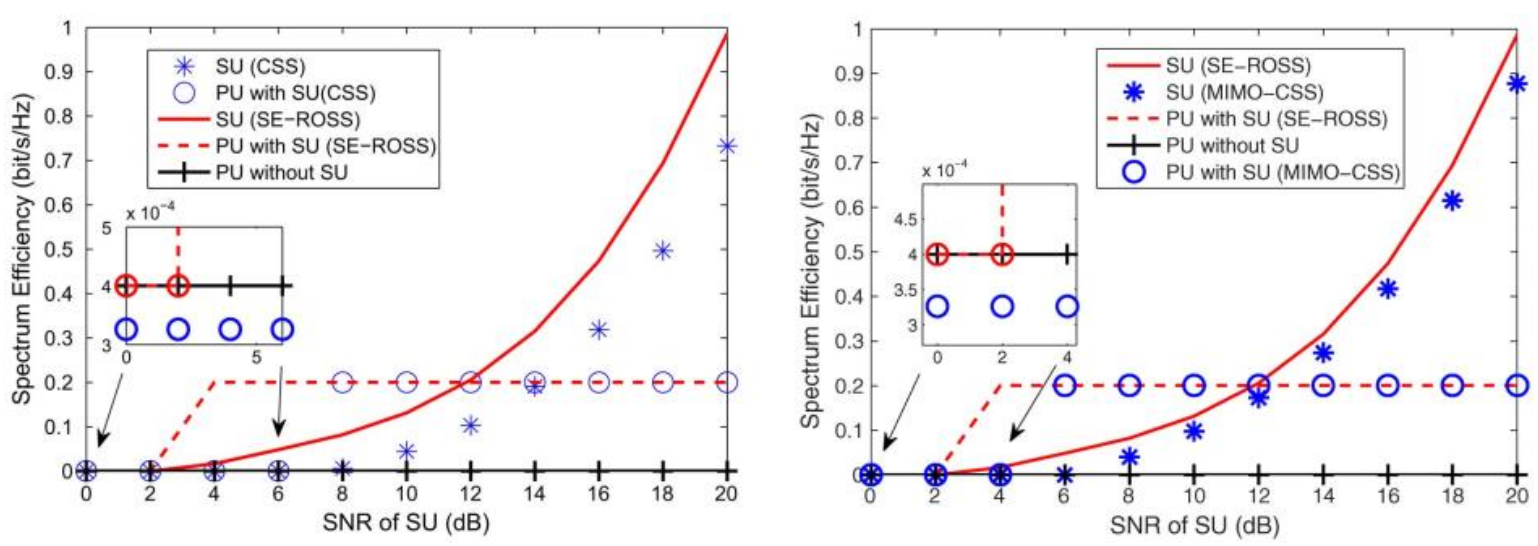

Figure 1. Simulations carried out comparing CSS and SE-ROSS (left) and MIMO-CSS and SE-ROSS (right) when the CSI is in perfect condition [34]

This simulation reveals that the spectral efficiency for the PU with SE-ROSS (compared to the other two systems) improves from a low condition of the SNR of the SU. The spectral efficiency increases for $\mathrm{SNR}=2 \mathrm{~dB}$ while this increase is seen in CSS for an $\mathrm{SNR}=6 \mathrm{~dB}$ and in MIMO-CSS for an $\mathrm{SNR}=4 \mathrm{~dB}$. Furthermore, the spectral efficiency for the secondary user also improves. For instance, the spectral efficiency is $1 \mathrm{bit} / \mathrm{s} / \mathrm{Hz}$ at an $\mathrm{SNR}=20 \mathrm{~dB}$ while it is approximately $0.73 \mathrm{bit} / \mathrm{s} / \mathrm{Hz}$ in CSS and close to $0.89 \mathrm{bit} / \mathrm{s} / \mathrm{Hz}$ in MIMO-CSS. Finally, the results lead to conclude that at least in simulations, the ROSS proposal can become a great alternative for spectrum sharing since it can allow the SU to generate traffic in a licensed channel. It is also beneficial so that the PU can reach the farthest PR.

\subsection{Spectrum sharing in mobile networks}

Currently, mobile networks are useful for everyday activities on a global scale and have suffered many transformations since the radioelectric spectrum began to be used for communications. There are 5 generations in current mobile networks (from $1 \mathrm{G}$ to $5 \mathrm{G}$ ) [35, 36], where each generation has brought improvements to quality of service (QoS), access to internet, short message services (SMS), among others. However, in spite of common line of thinking, each new generation does not replace the previous one completely. For instance, in Colombia, although fourth generation $(4 \mathrm{G})$ networks are available, there are still second and third generations ( $2 \mathrm{G}$ and $3 \mathrm{G}$ ) in many parts of the country. However, this is also related to the use of these networks in each area. Usually, these networks are located in remote areas far from cities where mobile networks are only used for cellphone calls. Furthermore, 2G and 3G networks have a wide coverage which is beneficial in rural zones where there are less users per square kilometer and the required network capacity is not significant.

Following up with the cellphone generation topic, although new generations do not replace the previous ones, the latter are less used over time given that the devices evolve and offer connectivity with the new mobile generations. Hence, $2 \mathrm{G}$ and $3 \mathrm{G}$ networks can be helpful to build cognitive radio networks and contribute to spectrum sharing. In [37], it is proposed that these mobile networks that are being less used could be reused to generate a new paradigm in spectrum sharing. This new technique of spectrum sharing has the capacity to support different generations of cellphone networks that operate on the same radio spectrum. The reuse of the spectrum or Spectrum Refarming (SR) is a proposal for mobile operators that intends to compensate for spectrum scarcity in these networks instead of focusing less on the cost benefits. For instance, when LTE reuses the GSM bands in the downlink, the orthogonal frequency division multiple access (OFDMA) can use the vacant GSM bands using the overlayer scheme.

This case study is based on the underlayer or subjacent SR scheme along with an OFDMA system to collect CDMA spectrum in a smart manner, harnessing the interference margin offered by CDMA. It works by considering a single cell with a CDMA sequence in the uplink in which the users are assigned at random with their propagation codes. When the comparison filter (MF) or minimum mean square error (MSE) [38] are applied, it is proven that when the propagation gain and the number of users grows large, the signal-plus-interference-noise-ratio (SINR) in the receiver output can converge to an SINR limit which is independent from the propagation codes, yet it is related to the CDMA system load defined as the load of the total number of active users under the propagation gain. In other words, for each received power, the maximum CDMA load can be quantified based on the SINR ranges. Hence, when the system operates under low levels of load, each user can suffer less interference and can tolerate a certain interference margin 
thus being able to handle the SINR ranges. This interference margin defines the maximum interference which can be introduced by the OFDMA system to the CDMA system in the subjacent SR system.

Finally, the proposed simulations for this scheme evidenced that it is possible to maintain the communication of the secondary user under the interference range of the primary user and thus avoid affecting transmission. This is achieved since the CDMA system sends its parameters to the OFDMA system to predict the interference margin. This is the only information needed between the systems to share the spectrum. Figure 2 shows a comparison between MF and MMSE.

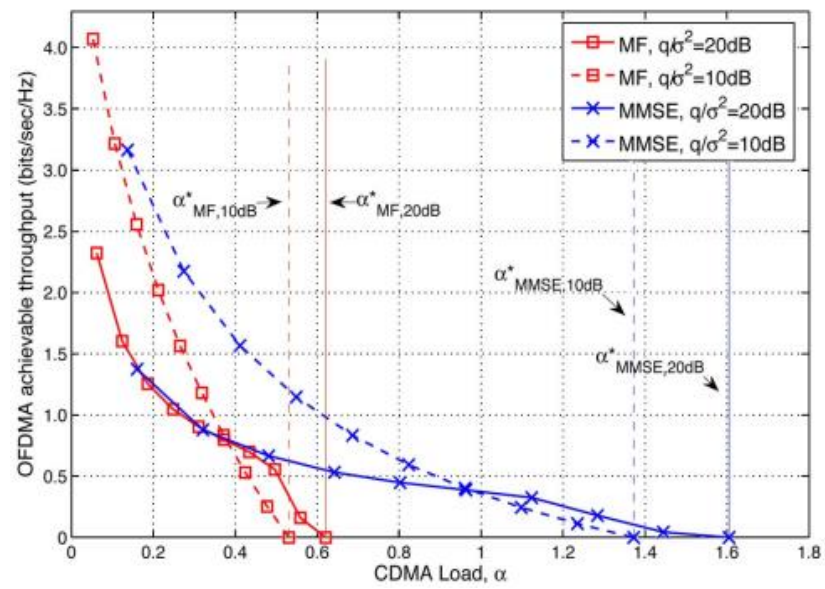

Figure 2. Throughput with OFDMA vs. CDMA load [37]

The analysis on Figure 2 evidenced that there is an improvement in throughput for the system for MMSE when the CDMA system load is increased compared to MF. As detailed in [37], it is explained that the CDMA system with the MMSE receiver can provide a high interference margin compared to MF. For simulations, two CDMA levels were considered to receive SNR. $20 \mathrm{~dB}$ and $10 \mathrm{~dB}$ were used to represent high and low reception power of CDMA. When the CDMA load is low, the OFDMA system can achieve greater performance and, when the load is high, there is low throughput for OFDMA. As the reception of SNR grows higher, CDMA will offer a greater interference margin while imposing more interference to OFDMA users.

New generation networks (NGN) [39] are networks where there is no link door to establish communication between equipment since they are in charge of defining their own network parameters for communication and information handover between devices. Similarly, this idea can be adopted in cognitive radio mobile networks to share the spectrum since in these types of networks, there is a base station that manages spectrum resources in the coverage area. Hence, the authors in [40] propose a network in which device-to-device (D2D) communication does not require information of the available spectrum from the base station since the equipment are in charge of establishing this connection.

For this spectrum sharing method, the orthogonal multiple access (OMA) and non-orthogonal multiple access (NOMA) schemes are proposed. In the first one, users must access the radio electric spectrum one at a time through resource blocks (RBs) while, in the second one, cellphone users and D2D users can reuse RBs which can cause interferences. It is then necessary to share the spectrum efficiently while also improving the performance of the network by taking into account the waiting queues during transmission.

In the proposed method for spectrum sharing, the simulation results were shown compared to the average length of the waiting queue and the average energy consumption. This revealed that there is a significant difference between the OMA and NOMA strategies since NOMA achieves lower delays and average values in the waiting queues compared to OMA. Furthermore. The energy consumption is also improved with spectrum sharing given that static OMA and NOMA have a better consumption compared to their dynamic and heuristic dynamic counterparts over the same systems as seen in Figure 3 . V is a control parameter that changes the priority of user energy consumption depending on the queue congestion.

Finally, the different schemes proposed offer a low complexity solution without requiring the channel and the probabilities of the information arrival process. Simulations also show that dynamic systems reduced the average of waiting queues in comparison to static methods for the same energy consumption. Additionally, NOMA surpasses the performance of OMA. 


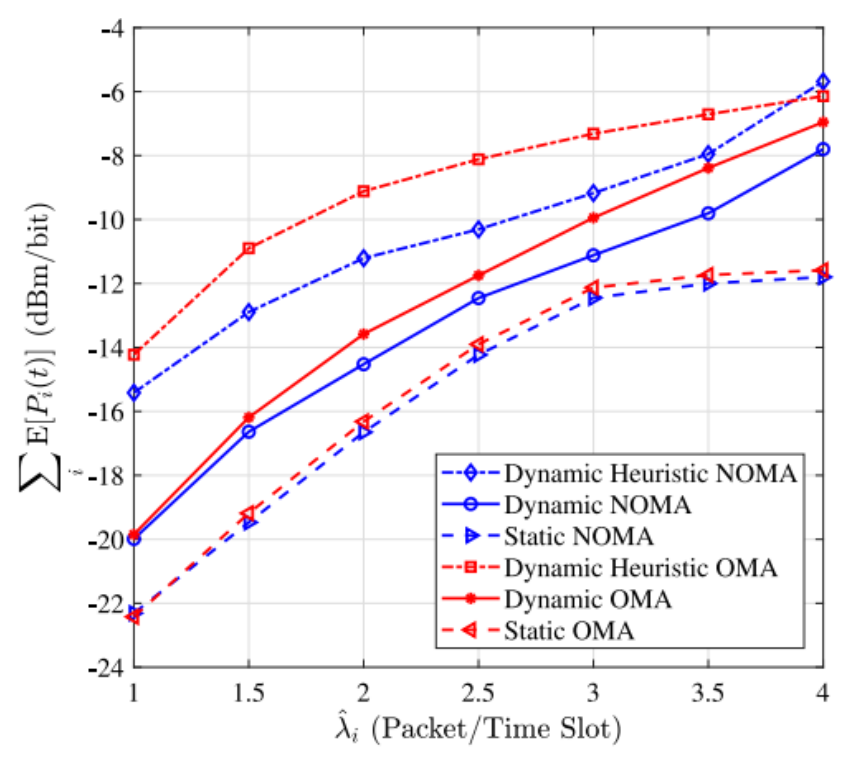

Figure 3. Total average consumption VS average arrival rate of information with $\mathrm{V}=100$

In mobile communications, the radioelectric spectrum is used by different operators depending on the regulations of each country. Hence, the spectrum can be shared between the same operators to tackle spectrum scarcity which may arise depending on the network status. It is necessary that the operators are in some way dynamic to adapt to different bandwidth requirements. In [41], the proximal spectrum access (PSA), as its name implies, enables the user in the closest base station regardless of the mobile service provider. This is an advantage for both the user and the provider since the latter can improve the limitations imposed upon him during spectrum leasing with the corresponding authority of each country and offer better quality in user experience.

The proposed system does not require significant changes. Therefore, the user equipment (UE) can request access to the closest base station (BS) and the latter can accept or reject it based on the information of the system load. In case this occurs, the UE can request access to any other proximal base station (PBS). This system includes a similar function to the roaming function used by operators when one of them has no coverage in the area where the user is located.

In the study carried out in [41] for the proposed system, simulations were carried out with GPS information of the base stations with the OpenCellID program which is a community that collects these type of data. Once this information has been obtained, different metrics were studied in various scenarios. The first metric involved the study of the average quality of experience (AQoE) [42] and, in the first scenario, user equipment are connected to the base station as long as there are enough resource blocks. A user equipment is considered to be blocked when the base station cannot assign a resource block. For the second scenario, the user equipment is not blocked and the resource blocks of the base station are assigned through the proportionally fair method. The second metric is the AQoE improvement using the association based on PSA (PSAbA) compared to the AQoE using the association based on the mobile network operator (MNObA). The third metric is the efficiency in the use of block resources which indicates the reduction of the allocation of the RB required by the base station to improve AQoE in PSAbA and MONbA. Finally, the last metric is the association factor X of PSA (PSAAFx) which reflects the decision of the user equipment for PSAbA when the RBs of the proximal base station are required by a service close to " $\mathrm{x}$ " percentage of the RBs of the mobile operator base station.

In this proposal, PSA shows an efficient use of BS for user download, which proves that there is an efficient spectrum exchange between operators. Different simulations carried out with the actual parameters of a mobile network showed that spectral resources to enable services are reduced by up to $46 \%$ in addition to the improvement of QoE for end users by up to 32\%. In the simulations in dense distributions, the reduction of resources was lower although the improvement of QoE was higher.

\subsection{Spectrum sharing in fifth generation resources $(5 G)$}

Fifth generation $(5 \mathrm{G})$ networks are the new strategy in mobile communications to allow higher upload and download speeds. However, in addition to this, recent networks led to the rise of the internet of things (IoT) [43] and improve the quality of service (QoS) for users. Since it is expected that this network is 
a better option compared to previous generations, it is also necessary to carry out studies regarding spectrum sharing given that networks are affected by spectrum scarcity due to the large number of devices connected to said network.

In 5G [44], a study is performed to implement spectrum sharing in 5G networks for unattended areas of the country. The proposal uses cloud-based spectrum sharing using OpenStack servers and the information collected by different sensors specialized in spectrum sensing. The server contains the information on the number of requirements of the system and obtain an estimation of the number of users in the network. With the collected data, the server has the capacity to analyze large amounts of information on spectrum use in $5 \mathrm{G}$ networks and generate paths to send information to the most remote places by using blank television bands.

Smart spectrum sharing based on wireless bandwidth networks (S3-WBN) serve as a medium to enable dynamic spectrum access (DSA) which was discussed in a previous section. Hence, it is possible to combine spectrum sharing and 5G networks. The proposal uses the software architecture known as simple sloppy semantic database (S3DB) [45]. One of the characteristics of this software architecture is the capacity to attend 35.000 clients simultaneously without discarding any package. The second characteristic is that all 35.000 requests must be attended in less than 15 seconds.

In simulations, 7 computers were used which carried out 5.000 queries each leading to 35.000 requests in 13 seconds without information losses using S3DB. The proposal reveals that spectrum sharing must also be accompanied by other areas of study such as cloud-based servers and also reach remote areas all over the world through sensors that collect information on the available spectrum in each area.

\subsection{Spectrum sharing in TV networks}

The management of the spectrum has evolved along with the digital conversion of television since it occupies a smaller bandwidth in the channels and offers the possibility of increasing the number of users that can harness these available channels. This has also been an advantage for television operators since simultaneous transmissions are now possible where only one transmission took place over the same channel. In these television bands, it is possible to generate a mutual leasing or a limited pool of the spectrum. In the first method, operators have licenses for the exclusive use of frequency bands, leasing parts of this spectrum to their peers when required while the second method includes a common pool of resources shared by various operators equitably.

Spectrum sharing in TV bands have to consider that these are mainly thought for rural areas with limited access or low number of users per square kilometer. In these areas, mobile networks and internet services are unable to reach users through guided medium or the coverage of mobile operators. As previously discussed, 5G networks are also intended to operate in these scenarios. Hence, it is uncommon to think of spectrum sharing in TV bands in the main cities given that there are few channels available to be used by secondary users.

In [46], a prototype of cognitive radio is proposed for spectrum sharing in TV white spaces so that secondary users can make use of them without affecting the communication of the primary user. This strategy was implemented in [47] which was called TV white spaces (TVWS) where it operates as regular television while the data from secondary users is sent through the inactive TV channels. The prototype [46] in Figure 4 defines that the secondary receivers obtain the information regarding the availability of the channels without using primary users from a spectrum database and then synchronize with the available channel for transmission. Hence, there is no interference with the transmission of the TV stations. Figure 5 shows the transfer channel process when the primary user is active. The green arrow refers to the actions of the CR-TV user, the red and blue arrows represent the secondary transmitter and receiver respectively.

When the user switches to another channel, the database is updated with this information and CR-TV users notice the change and move to transmit over another available channel. The database is updated once again with the information from the secondary user. The secondary receiver receives the new channel for transmission and performs the change in the secondary equipment so that transmission is maintained.

\subsection{Concepts and business ideas}

As mentioned beforehand, due to the number of users required to use the radioelectric spectrum in different frequency channels, it is required that some licensed bands are shared and increase the connection capacity. In terms of spectrum sharing, it is forecast that the number of subscribers for 2020 will grow to 5.7 billion with a penetration rate of $73 \%$ and, in terms of SIM device connection, it is expected that 9.7 billion devices are connected with a penetration range of $112 \%$ [48].

Spectrum sharing can also bring benefits such as allowing more users to use the licensed bands and improve the quality of service (QoS) for different needs related to content and live communications. This wider commercial offer leads to market competition, network investment to increase connection 
capabilities and reduction in costs. Spectrum sharing can translate into benefits for the operators but must also consider that it is not possible to generalize spectrum sharing since it will be different depending on the areas where it is implemented. For instance, the same strategy cannot be used in the center of a city where a large number of users gathers and in the edges of said city where the spectrum requirement per user is low. Therefore, a regulatory frame is necessary where operators and users can contemplate the investment possibilities within this paradigm to bring a larger number of benefits from this resource.

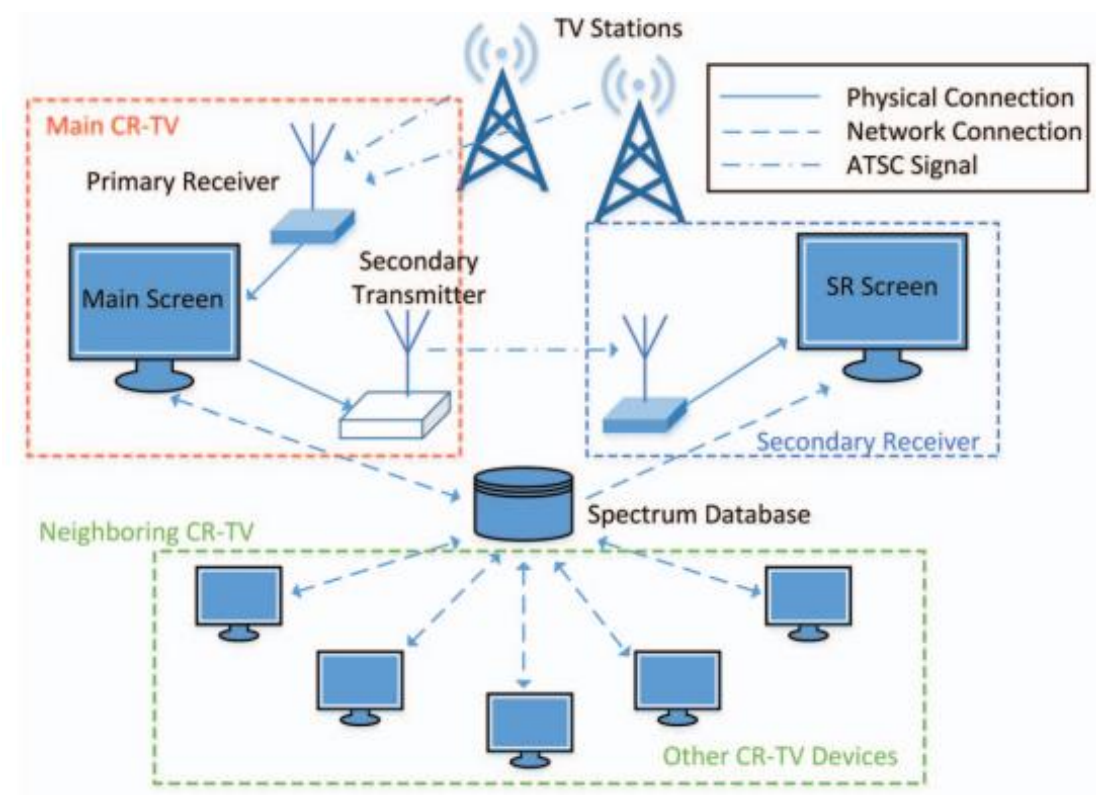

Figure 4. Prototype of the cognitive radio TV system

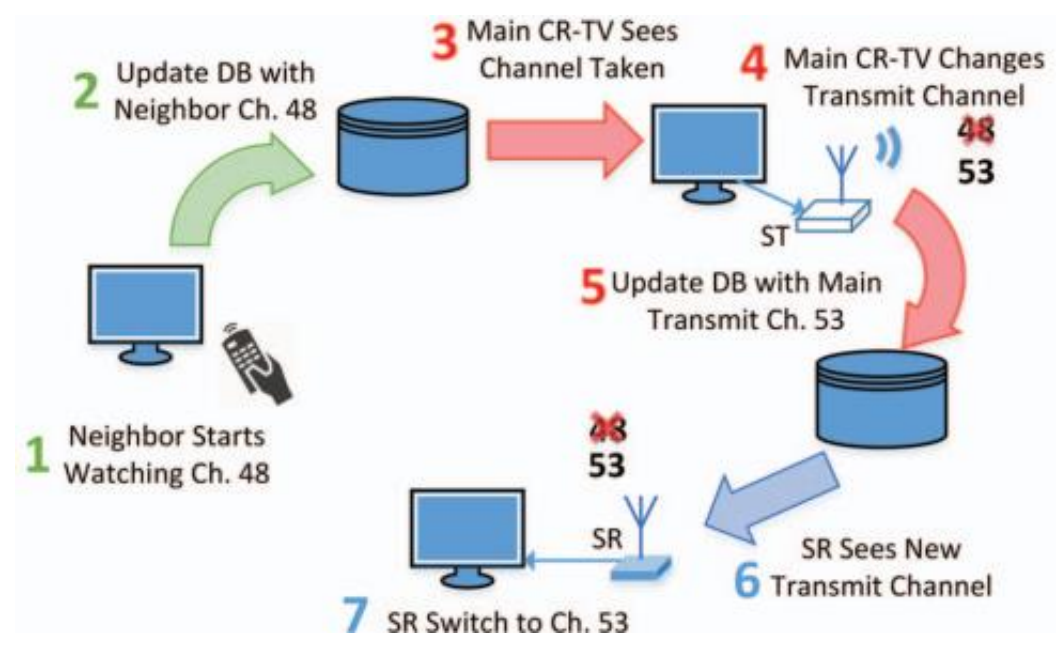

Figure 5. Example of a transfer process when the CR-TV user changes channel

\section{DISCUSSION}

Finally, as shown in the article, there are many proposals in terms of spectrum sharing and efforts are still made in order to improve how these resources are managed. Hence, it is necessary to keep on working on these aspects and put into action different strategies to determine which are most suitable, keeping in mind the legislations for each country or place where this technology is required. Additionally, the type of place where the spectrum is shared needs to be considered given that it is not the same scenario in highly-populated cities or capital cities than in rural areas due to the intrinsic resource demand. 
Licensed users should be included in the solution since it is them who will be benefitted from sharing the spectrum both in terms of the efficiency levels of the system and the increased coverage in areas distanced from urban perimeters. In the spectrum used for TV along with $5 \mathrm{G}$ networks, many opportunities lie ahead to keep implementing spectrum sharing. Satellite networks must also be considered since they can share the spectrum and improve the coverage of existing networks along with licensed users. Table 1 shows a comparative summary of the discussed strategies in addition to the possible improvements that each one may include.

Table 1. Comparative analysis between the studied strategies in terms of their advantages, disadvantages and possible improvements

\begin{tabular}{|c|c|c|c|c|}
\hline $\begin{array}{l}\text { Spectrum sharing } \\
\text { strategies }\end{array}$ & Description & Advantages & Disadvantages & Possible Improvements \\
\hline Cooperative & $\begin{array}{l}\text { The spectrum can } \\
\text { be shared as long as } \\
\text { there is cooperation } \\
\text { with the primary } \\
\text { user. }\end{array}$ & $\begin{array}{l}\text { The primary user } \\
\text { receives help from the } \\
\text { secondary users in } \\
\text { exchange of sharing } \\
\text { the spectrum. }\end{array}$ & $\begin{array}{l}\text { The network must be } \\
\text { properly configurated to } \\
\text { avoid interferences. } \\
\text { Although the interference- } \\
\text { free communication is } \\
\text { possible as seen in. }\end{array}$ & $\begin{array}{l}\text { Study cooperative spectrum } \\
\text { sharing in practice and determine } \\
\text { whether it is a suitable alternative } \\
\text { for cooperative spectrum sharing. }\end{array}$ \\
\hline Mobile & $\begin{array}{l}\text { Mobile resources } \\
\text { are shared by } \\
\text { mobile operators. }\end{array}$ & $\begin{array}{l}\text { Mobile operators can } \\
\text { share the spectrum } \\
\text { even among } \\
\text { competitors when } \\
\text { needed. }\end{array}$ & $\begin{array}{l}\text { Operators can charge to } \\
\text { share the spectrum with } \\
\text { secondary users and even } \\
\text { establish a monopole in } \\
\text { the acquire bands. }\end{array}$ & $\begin{array}{l}\text { Carry out a study on the charges } \\
\text { that spectrum sharing could } \\
\text { generate in mobile operators. }\end{array}$ \\
\hline $5 \mathrm{G}$ & $\begin{array}{l}\text { It is the next } \\
\text { generation of } \\
\text { mobile networks. }\end{array}$ & $\begin{array}{l}\text { It can use TV bands } \\
\text { to reach distanced } \\
\text { areas. }\end{array}$ & $\begin{array}{l}\text { It is a system that is still } \\
\text { being placed. Hence, it } \\
\text { can lead to changes in } \\
\text { spectrum sharing in this } \\
\text { type of networks. }\end{array}$ & $\begin{array}{l}\text { Given that this technology is still } \\
\text { in development, more studies on } \\
\text { how to share spectrum in new } \\
\text { networks could be ideal. }\end{array}$ \\
\hline TV & $\begin{array}{l}\text { Radioelectric } \\
\text { spectrum with free } \\
\text { bands due to the } \\
\text { migration from } \\
\text { analog to digital. }\end{array}$ & $\begin{array}{l}\text { It has the possibility } \\
\text { of reaching remote } \\
\text { areas where no other } \\
\text { topologies have } \\
\text { access. }\end{array}$ & $\begin{array}{l}\text { The analog shutdown } \\
\text { must take place before } \\
\text { implementing the } \\
\text { spectrum sharing. }\end{array}$ & $\begin{array}{l}\text { Carry out a study on the } \\
\text { advantages that spectrum sharing } \\
\text { could have in remote places and } \\
\text { whether the technologies to } \\
\text { receive these types of networks are } \\
\text { in place. }\end{array}$ \\
\hline
\end{tabular}

\section{CONCLUSION}

Cognitive radio networks must undoubtedly become a part of future generations of wireless networks that are being developed. As mentioned at the beginning of the article, significant scarcity is present in the radioelectric spectrum given the large number of users that require access. This is where spectrum sharing comes in with different schemes and processes that can be carried out so that more devices can have access to the spectrum without interfering with the communications of licensed users. These studies are only a small part of what has been achieved in spectrum sharing. Hence, there are many topics to work on, even those that are rarely analyzed such as spectrum sharing in satellite networks. This article focused on a general overview of the different strategies proposed for spectrum sharing and TV and mobile networks finding significant progress and simulations, yet it has not been possible to put these solutions into practice to solve spectrum scarcity.

There is still work ahead in spectrum sharing proposals as well as simulations and, most importantly, the practice in different strategies that are proposed. However, the regulations of each country could restrict the access to spectrum resources yet it is a step that needs to be taken to optimize the use of wireless networks. Fifth generation networks must come closer to cognitive radio systems with new proposals given that they are the next step in terms of mobile networks and many other services that are currently available or that may occur in the future such as the Internet of Things (IoT).

\section{ACKNOWLEDGEMENTS}

The researchers would like to acknowledge the support and funding of this research project by the Center for Research and Scientific Development of Universidad Distrital Francisco José de Caldas.

\section{REFERENCES}

[1] C. Hernández, et al., "MCDM Spectrum Handover Models for Cognitive Wireless Networks," World Academic of Science, Engineering and Technology, International Journal of Electronics and Communication Engineering, vol. 9 , no. 10 , pp. 1039-1042, 2015. 
[2] M. Tuberquia-David, et al., "A multifractal wavelet model for the generation of long-range dependency traffic traces with adjustable parameters," Expert Systems with Applications, vol. 62, pp. 373-384, 2016.

[3] C. A. H. Suarez, et al., "Fuzzy Feedback Algorithm for the Spectral Handoff in Cognitive Radio Networks," Revista Faculted de Ingenieria, vol. 81, pp. 47-62, 2016.

[4] C. Hernández, et al., "Algoritmos para asignación de espectro en redes de radio cognitiva," Tecnura, vol. 20, no. 48 , pp. 69-88, 2016.

[5] C. A. H. Suarez, et al., "Modelo adaptativo multivariable de handoff espectral para incrementar el desempeño en redes móviles de radio cognitiva,” Bogotá, Editorial UD, 2017.

[6] J. Mitola and G. Q. Maguire Jr., "Cognitive radio: making software radios more personal," IEEE Personal Communications, vol. 6, no. 4, pp. 13-18, 1999.

[7] C. Hernández, et al., "Multivariable algorithm for dynamic channel selection in cognitive radio networks," EURASIP Journal on Wireless Communications and Networking, vol. 2015, no. 1, pp. 1-17, 2015.

[8] H. Vasquez, et al., "Proactive Spectrum Handoff Model with Time Series Prediction," International Journal of Applied Engineering Research, vol. 10, no. 21, pp. 42259-42264, 2015.

[9] H. Kour, et al., "A comprehensive survey on spectrum sharing: Architecture, energy efficiency and security issues," Journal of Network and Computer Applications, vol. 103, pp. 29-57, 2018.

[10] C. Yang, et al., "Advanced spectrum sharing in 5G cognitive heterogeneous networks," IEEE Wireless Communications, vol. 23, np.2, pp. 94-101, 2016.

[11] P. S. Yawada and M. T. Dong, "Intelligent Process of Spectrum Handoff/Mobility in Cognitive Radio Networks," Journal of Electrical and Computer Engineering, vol. 2019, pp. 1-12, $20[$.

[12] K. Kumar, et al., "Spectrum handoff in cognitive radio networks: A classification and comprehensive survey," Journal of Network and Computer Applications, vol. 61, pp. 161-188, 2016.

[13] Y. S. Chen and J. S. Hong, "A Relay-Assisted Protocol for Spectrum Mobility and Handover in Cognitive LTE Networks," IEEE Systems Journal, vol. 7, no. 1, pp. 77-91, 2013.

[14] S. Gourdache, et al., "A framework for spectrum harvesting in heterogeneous wireless networks integration," Journal of King Saud University - Computer and Information Science, 2019.

[15] L. Hu, et al., "Optimal energy-efficient transmission for hybrid spectrum sharing in cooperative cognitive radio networks," China Communications, vol. 16, no. 6, pp. 150-161, 2019

[16] L. Gao, et al., "Spectrum Trading in Cognitive Radio Networks: A Contract-Theoretic Modeling Approach," IEEE Journal on Selected Areas in Communications, vol. 29, no. 4, pp. 843-855, 2011.

[17] J. Gazda, et al., "Dynamic spectrum leasing and retail pricing using an experimental economy," Computer Networks, vol. 121, pp. 173-184, 2017.

[18] I. F. Akyildiz, et al., "NeXt generation/dynamic spectrum access/cognitive radio wireless networks: A survey," Comput. Networks, vol. 50, no. 13, pp. 2127-2159, 2006.

[19] Q. Zhao and B. M. Sadler, “A Survey of Dynamic Spectrum Access," IEEE Signal Process Magazine, vol. 24, no. 3, pp. 79-89, 2007.

[20] C. Hernández and D. A. Giral, "Spectrum Mobility Analytical Tool for Cognitive Wireless Networks," International Journal of Applied Engineering Research, vol. 10, no. 21, pp. 42265-42274, 2015.

[21] W. Lehr and J. Crowcroft, "Managing shared access to a spectrum commons," in First IEEE International Symposium on New Frontiers in Dynamic Spectrum Access Networks, 2005. DySPAN 2005., pp. 420-444, 2005.

[22] W. Liang, et al., "Cooperative Overlay Spectrum Access in Cognitive Radio Networks," IEEE Communications Survey and Tutorials, vol. 19, no. 3, pp. 1924-1944, 2017.

[23] M. El Tanab and W. Hamouda, "Resource Allocation for Underlay Cognitive Radio Networks: A Survey," IEEE Communications Survey and Tutorials, vol. 19, no. 2, pp. 1249-1276, 2017.

[24] S. Geirhofer, et al., "Cognitive Radios for Dynamic Spectrum Access - Dynamic Spectrum Access in the Time Domain: Modeling and Exploiting White Space," IEEE Communications Magazine, vol. 45, no. 5, pp. 66-72, 2007.

[25] F. Akhtar, et al., "White space: Definitional perspectives and their role in exploiting spectrum opportunities," Telecommunications Policy, vol. 40, no. 4, pp. 319-331, 2016.

[26] L. Giupponi and C. Ibars, "Distributed cooperation in cognitive radio networks: Overlay versus underlay paradigm," IEEE Vehicular Technology Conference, pp. 1-6, 2009.

[27] S. M. Baby and M. James, "A Comparative Study on Various Spectrum Sharing Techniques," Procedia Technology, vol. 25, pp. 613-620, 2016.

[28] S. S. Nair, et al., "Hybrid spectrum sharing in dynamic spectrum access networks," in The International Conference on Information Networking 2013 (ICOIN), pp. 324-329, 2013.

[29] M. F. Kader, et al., "Cooperative spectrum sharing in cognitive radio networks: An interference free approach," Physical Communication, vol. 25, no. 1, pp. 66-74, 2017.

[30] R. Y. Mesleh, et al., "Spatial modulation," IEEE Transactions on Vehicular Technology, vol. 57, no. 4, pp. 2228-2241, 2008.

[31] R. Mesleh, et al., "Spatial Modulation - A New Low Complexity Spectral Efficiency Enhancing Technique," in 2006 First International Conference on Communications and Networking in China, pp. 1-5, 2006.

[32] S. Tang and B. L. Mark, "Performance Analysis of a Wireless Network with Opportunistic Spectrum Sharing," in IEEE GLOBECOM 2007 - IEEE Global Telecommunications Conference, pp. 4636-4640, 2007.

[33] L. Duan, et al., "Cooperative Spectrum Sharing: A Contract-Based Approach," IEEE Transactions on Mobile Computing, vol. 13, no. 1, pp. 174-187, 2014. 
[34] R. Zhu, et al., "Relay Opportunistic Spectrum Sharing Based on the Full-Duplex Transceiver," IEEE Transactions on Vehicular Technology, vol. 64, no. 12, pp. 5789-5803, 2015.

[35] T. Mshvidobadze, "Evolution mobile wireless communication and LTE networks," 2012 6th International Conference on Application of Information and Communication Technologies (AICT 2012), pp. 4-10, 2012.

[36] A. Gupta and R. K. Jha, "A Survey of 5G Network: Architecture and Emerging Technologies," IEEE Access, vol. 3, pp. 1206-1232, 2015.

[37] S. Han, et al., "Spectrum refarming: A new paradigm of spectrum sharing for cellular networks," 2014 IEEE Global Communications Conference, vol. 63, no. 5, pp. 893-898, 2014.

[38] C. Hernández, O. Salcedo, A. Escobar. "An ARIMA model for forecasting Wi-Fi data networks traffic values", Revista Ingeniería e Investigacion, Vol. 29, pp. 65-69, 2009.

[39] C. Hernández, D. Lopez, D. Giral. "Modelo de decisión espectral colaborativo para mejorar el desempeño de las redes de radio cognitiva", Primera. Bogotá: Editorial UD, 2020.

[40] M. Kazeminia, et al., "Delay-aware spectrum sharing solutions for mixed cellular and D2D links," Computer Communications, vol. 139, pp. 58-66, 2019.

[41] M. Srinivasan and C. S. R. Murthy, "Proximal spectrum access: A QoE-driven inter-operator spectrum sharing paradigm," IEEE International Symposium on Personal. Indoor and Mobile Radio Communications (PIMRC), pp. 1764-1768, 2015.

[42] K. Kilkki, "Quality of experience in communications ecosystem," Journal of Universal Computer Science, vol. 14, no. 5, pp. 615-624, 2008.

[43] B. Jamoussi, "IoT Prospects of Worldwide Development and Current Global Circumstances," Presentation Slides, Telecommunication Standardization Bureau, ITU, 2010.

[44] L. Montsi, et al., "A real-time big-data system for smart spectrum sharing: Enabling affordable broadband in $5 \mathrm{~g}$ for underserved communities," 2017 Global Wireless Summit, (GWS 2017), pp. 245-251, 2017.

[45] "Simple Sloppy Semantic Database," Wikipedia.

[46] D. Rempe, et al., "A cognitive radio TV prototype for effective TV spectrum sharing," 2017 IEEE International Symposium on Dynamic Spectrum Access Networks, (DySPAN 2017), pp. 1-2, 2017.

[47] D. Pineda, C. Hernández. "Cognitive radio for TVWS usage", TELKOMNIKA Telecommunication Computing Electronics and Control, Vol. 17, No. 6, pp. 2735-2746, 2019.

[48] L. Gallitto, "Compartición de Espectro: Impactos y Oportunidades para el Futuro," 2017. [Online]. Available: https://www.itu.int/en/ITU-R/seminars/rrs/RRS-17-Americas/Documents/Forum/4_GSMA_Lucas\%20Gallito.pdf. 